\title{
Numerical Study of the Thermal Efficiency of a School Building with Complex Topology for Different Orientations
}

\author{
Eusébio Z.E. Conceição ${ }^{1} \quad M^{\mathrm{a}}$ Manuela J.R. Lúcio ${ }^{2}$ \\ ${ }^{1}$ FCMA - University of Algarve - Campus de Gambelas - 8005-139 Faro, Portugal \\ ${ }^{2}$ Vertical Grouping of Schools Professor Paula Nogueira/Regional Directory of Education \\ of Algarve, Rua da Comunidade Lusíada, 8700-248 Olhão, Portugal
}

\section{Key Words}

Numerical simulation - building thermal behavior . complex topology · warm environments $\cdot$ thermal comfort . PMV index

\footnotetext{
Abstract

In this work a numerical model that simulates the thermal behavior of a building with complex topology and evaluates the indoor thermal and air quality, in transient conditions, is used for a school building thermal project. The program calculates the building surfaces solar radiation field, the building's temperatures, the internal environmental variables, and the occupant's comfort levels.

Initially, after the numerical model is validated, the software is used to evaluate the school building's thermal response for four different orientations, either in winter or summer conditions. The work then aims to identify uncomfortable spaces in order to propose, as an example, several solutions that could be introduced for each orientation, that would improve the thermal
}

comfort and air quality levels to which the occupants are subjected, and decrease the building's energy consumption levels. The information obtained from this study could be used to help a designer choose which thermal systems and solutions function best for a preferred school building orientation.

\section{Introduction}

In order to evaluate the thermal comfort level in a moderate environment equipped with an air-conditioning system, in either a cold or in a warm climate during winter or summer, the PMV (Predicted Mean Vote) and the PPD (Predicted Percentage of Dissatisfied) indexes are used [1-3]. The PMV index is given as a value on the seven-point comfort scale ( -3 cold; -2 cool; -1 slightly cool; 0 neutral; 1 slightly warm; 2 warm, and 3 hot) and is based on four environmental parameters (air mean temperature, velocity, relative humidity, and radiant mean temperature) and two personal factors (clothing and 
metabolic activity levels). In accordance with main-stream thinking about the PMV and PPD indexes, the thermal neutrality of an individual is obtained when the body's heat loss is equal to the body's metabolic heat $(\mathrm{PMV}=0)$.

The standards predict acceptable fluctuations in the comfort conditions because of the difficulties in obtaining thermal neutrality for all people that share the same compartment at the same time. For acceptable thermal comfort conditions, in accordance with ISO 7730 [2], the PMV should change between -0.5 and 0.5 , which means that the percentage of dissatisfied people is $<10 \%$. Nevertheless, more recently the CR 1752 [3] defines three comfort categories (A, B, and C), that establish limits for PMV and PPD indexes: the PMV index values in category A change between -0.2 and 0.2 (percentage of dissatisfied people $<6 \%$ ), in category $B$ between -0.5 and 0.5 (percentage of dissatisfied people $<10 \%$ ) and in category $\mathrm{C}$ between -0.7 and 0.7 (percentage of dissatisfied people $<15 \%$ ). This classification allows the selection a priori of one thermal environment according to the demands requested.

Fanger and Toftum [4] presented an extension of the PMV model, to be used in nonair-conditioned buildings in warm climates. This extension, used in warm environments, combines the "static" PMV model and the adaptive model. The idea was to use the traditional PMV model, which considered the thermal balance of the human body, and the expectations verified in the adaptive model (see also de Dear et al. [5]). The extension of the PMV model, to be used for people who are not subjected to air-conditioned environments, was based on an expectancy factor that could be multiplied by the "static" PMV value. The expectancy factor changed between 0.5 and 1 , with the value 1 used for airconditioned environments [4].

Studies of school buildings that have been made over recent years (for example [6-8]) show an interesting topic that can be analyzed and their ideas implemented. These kinds of studies, due to their importance, have been made in most countries throughout the world. They consider different climates and heating, ventilation, and airconditioning systems, that aim to evaluate and improve the comfort levels, namely the thermal comfort, the air quality, the visual comfort, the acoustical comfort and other comfort levels.

The thermal comfort level, that the students feel in classrooms with nonuniform environments, depends on personal parameters and the distribution around the body of the values of the following environmental variables: air temperature, air velocity, air relative humidity, radiant temperature, surfaces temperature, incident solar radiation, clothing temperature and humidity, and others. Some of these topics, numerically or experimentally analyzed, have been discussed more recently elsewhere [9-12].

In reality, the selection of thermal systems and thermal solutions to be implemented in different school building spaces are not made with regard to the criterion of occupants comfort, because information regarding the different orientation of the buildings are not available. In the present study the software created will be used to analyze four different building orientations, in winter or summer conditions, and to identify uncomfortable spaces in order to propose exemplary solutions for each orientation, that would improve the thermal comfort and air quality levels for occupants and decrease the building's energy consumption. The information obtained can then be used to help designers choose the best thermal systems and solutions to function in the preferred school building orientation.

To evaluate occupants thermal comfort levels, in a warm environment without air-conditioning installed inside compartments during summer conditions, the Fanger model [1] was used with extrapolation from Fanger and Toftum [4], nevertheless, in winter conditions, only the Fanger model [1] was used.

\section{Methodology}

\section{Building Thermal Response Numerical Model}

The numerical model that simulates the buildings' thermal behavior and which works in steady-state and transient conditions, was based on energy and mass balance integral equations (see details in Conceição and Conceição et al. [13-17]).

The energy balance integral equations for this work, were developed for the air (inside the several compartments), the different window glasses, the interior bodies (located inside the several compartments) and the different layers of the building's main structures, while the mass balance integral equations were developed for the water vapor (inside the several compartments) and air contaminants (inside the several compartments).

In the resolution of this system of equations, which in this work used transient conditions, the Runge-KuttaFehlberg [18] method with error control was used. The model considers conductive, convective, radiative, and mass transfer phenomena. The conduction was verified in the building's main structures (doors, ceiling, ground, 
walls, etc.) layers. For convection the natural, forced and mixed phenomena were considered, while for radiation, verified inside and outside the building, the short-wave (the real distribution of direct solar radiation in external and internal surfaces) and long-wave (heat exchanges between the building's external surfaces and the surrounding surfaces and among the internal surfaces of each compartment) phenomena were considered. In the radiative calculus, the shading effect caused by the surrounding surfaces and by the internal surfaces was considered.

\section{Occupant's Thermal Comfort Numerical Model}

The topic of the occupant's thermal comfort has been analyzed by several authors in recent years. In these studies empirical models that calculated the thermal comfort levels based on environmental values, experimentally obtained, were developed and used. Similarly, thermal dynamic models that numerically calculate the compartments thermal comfort mean value in buildings, computational fluids dynamics models that numerically calculate the environmental variables around the occupants and numerical and experimental manikins that evaluate the human thermal response based in thermoregulation principles [1,9,19-21].

In this work, to evaluate the thermal comfort level to which occupants are subjected, the PMV index [1-3] was used. In this calculus the personal parameters and the environmental parameters are considered. Initially the activity (1.2 Met) and clothing levels (1 Clo of clothing in winter conditions and 0.5 Clo of clothing in summer conditions) were considered, while secondly, the air mean temperature (numerically calculated for each compartment), the air mean relative humidity (numerically calculated for each compartment), the radiant mean temperature (obtained through the mean value of the temperatures of the compartment's surrounding surfaces) and the air mean velocity $\left(0.15 \mathrm{~m} \mathrm{~s}^{-1}\right.$ in winter conditions and $0.25 \mathrm{~m} \mathrm{~s}^{-1}$ in summer conditions) were considered.

\section{Simplified Model of a Building}

The modern school building analyzed, to be built in Southern Portugal, was divided into three floor levels: the first floor, the second floor, and the roof (Figure 1). Each floor was formed by different compartments; the building's main structures, the building's interior bodies, and glass in the windows. In relation to the main bodies all existing external bodies that promoted shading were also considered. In the roof, isolated from the second floor, glass was used in the upper part. This air collector could be used in the future to heat the air to be injected into cold compartments under winter conditions. This philosophy was not applied in this work, nevertheless, the air collector's influence on the building's thermal response was considered.

This modern school building, with three floor levels, was divided into 97 compartments with 1277 main structures, 233 glazed windows, and 272 interior bodies (desks).

The school building was divided into three blocks: Block 3 (the biggest) is to be used for lessons, Block 2 (medium sized) will also be used for lessons and Block 1 (the smallest) is to be used for the catering facilities and the administration (Figure 1).

In Figure 1, the grid generation used in the numerical simulation is presented. This numerical grid, used in the determination of internal and external direct solar radiation, was spaced $30 \mathrm{~cm}$ in both directions. This figure also shows the four analyzed referential layouts.

Each layout was associated with the orientation that could be chosen for the future school building to be built. Thus, in:

Layout 1- The biggest block (block 1) and the medium block (block 2) are oriented in the East-West direction and the smallest block (block 3 ) is placed East in relation to the bigger blocks;

Layout 2- The biggest block (block 1) and the medium block (block 2) are oriented in the SouthNorth direction and the smallest block (block 3) is placed South in relation to the bigger blocks;

Layout 3- The biggest block (block 1) and the medium block (block 2) are oriented in the East-West direction and the smallest block (block 3) is placed West in relation to the bigger blocks;

Layout 4- The biggest block (block 1) and the medium block (block 2) are oriented in the SouthNorth direction and the smallest block (block 3) is placed North in relation to the bigger blocks.

\section{Validation of the Numerical Model}

The numerical model to simulate the thermal response of a building with complex topology was validated in a school building similar to the one analyzed in this work (oriented as in layout 1), located in South Portugal (Algarve), on a winter and a summer day. For this validation, the doors and windows were closed, the airconditioning systems were turned off and the effect of indoor curtains and radiative heat exchanges were not considered. In these numerical simulations the air exchange rate inside each compartment, by infiltration, 


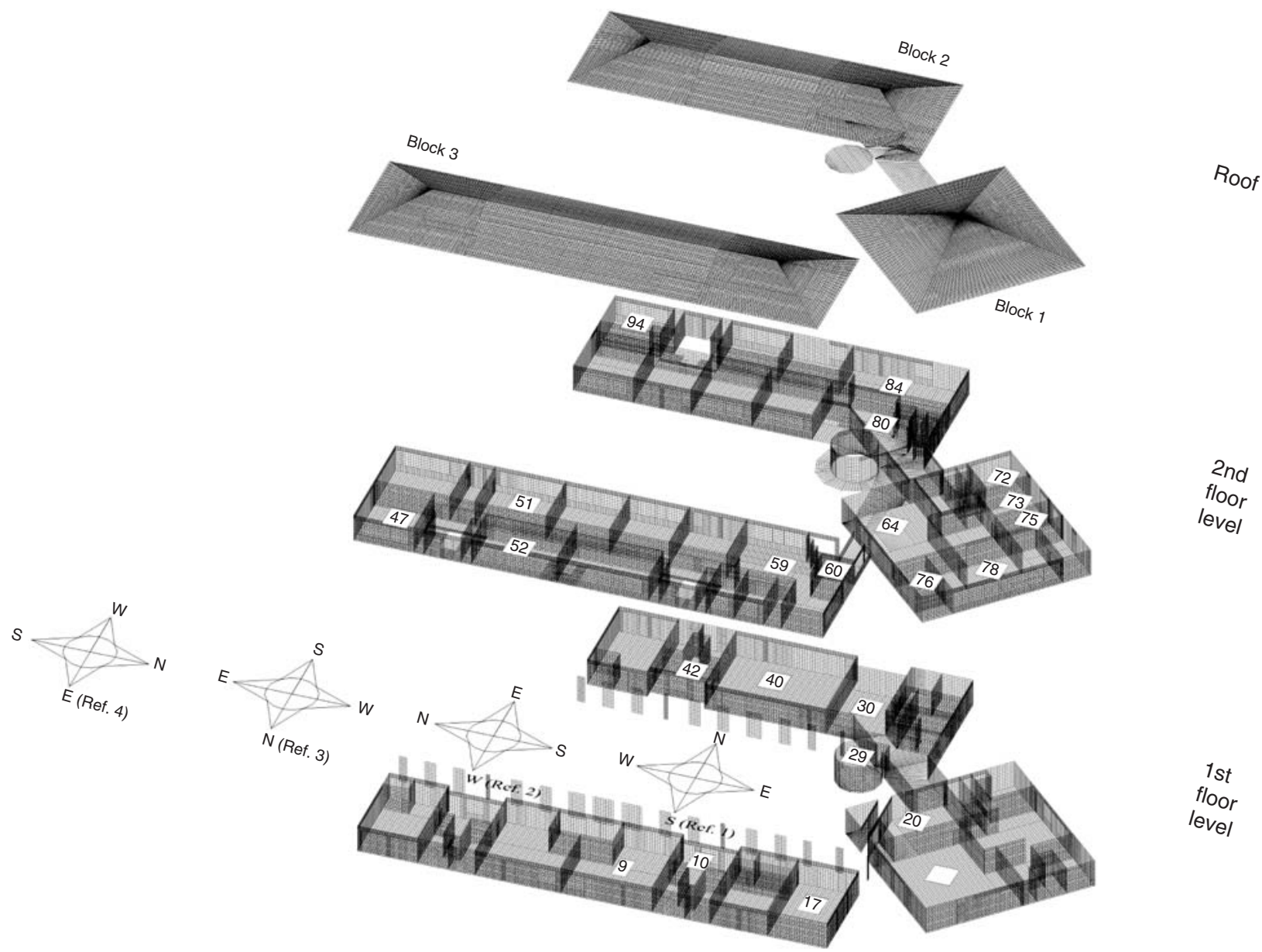

Fig. 1. Grid generation in the 3 levels of the school building and of the 4 analysed layouts, that are based on the geometry of the School EB 2,3 Poeta Emiliano da Costa, (Estoi, Faro).

were obtained experimentally, in several compartments, using the tracer gas concentration method.

Conceição and Lúcio [14] presented the validation of the numerical model for winter conditions. In conclusion, it was verified that the model reproduces the experimental values well. In general, the difference between numerical and experimental air temperature values is lower than $2^{\circ} \mathrm{C}$. A maximum difference of $4^{\circ} \mathrm{C}$ in compartments with windows turned towards the South was verified, but only during some hours in the afternoon. The difference between numerical and experimental air relative humidity values, in general, varied between 10 and $20 \%$. The highest value was verified in the first hours of the day.

Conceição and Lúcio [16] gave a validation of the numerical model for extreme conditions in summer. Measured and calculated results for the indoor air temperature were compared. The validation tests showed a good agreement between experimental and numerical values in all analyzed compartments. In general, the error verified was around $1^{\circ} \mathrm{C}$. Nevertheless, some discrepancies were shown, namely: the indoor air temperature experimental results, in general, were slightly higher than the numerical values. There was a time delay in the maximum temperature, but only in a few compartments, between experimental and numerical results, of around $3 \mathrm{~h}$ maximum. Also, there was a maximum air temperature difference between numerical values and experimental results, in general lower than $2^{\circ} \mathrm{C}$, found when the compartment's air temperature values were the highest; at the end of the afternoon (but only in some compartments). The temperature was lower in the experimental results and higher in the numerical values. These discrepancies were due to aspects associated with simplification factors. This illustrated that; overall, the control of these aspects in buildings with such characteristics is very complex. 


\section{Results and Discussion}

In this study a numerical model has been validated and used to evaluate the thermal response of a school building and the occupant's thermal comfort levels for four different orientations, either in winter or summer conditions. The results obtained were used to identify some examples of different thermal systems and thermal solutions for each orientation.

The input data, associated with the building's geometry, were used as numerical input to the model in the development of an integral equations system. In this simulation the numerical model was formed by 12870 integral equations, namely 97 for the air, 12074 for the main layers of the structures (1277 building's main structures), 233 for the windows, 272 for the interior structures, and 194 for the gases (water vapor and carbon dioxide).

The external environmental variables used as input data, namely the air temperature $(T)$, air relative humidity $(\mathrm{RH})$, air velocity $(V)$ and air direction $(D)$, were experimentally measured in the Algarve's central region. On a typical winter's day, the information measured on the 15th February was used (Figure 2), while on a typical summer day the information measured on 12th June was used (Figure 3), both in 2004. The initial temperatures for the buildings: structures and rooms, in accordance with different previous simulations, were $15^{\circ} \mathrm{C}$ for the winter day and $25^{\circ} \mathrm{C}$ for the summer day.

Due to the large number of compartments, it was decided for this study to analyze selected rooms, having taken into consideration the compartment location in the Block, compartment location in the floor levels, compartment volume, windows location and windows area. In Table 1, the characteristics of the selected typical rooms are presented (also see Figure 1).

In this work, a ventilation system that considered extraction fans installed in the different compartments and working between 8:30 a.m. and 18:45 p.m. was used. The philosophy considered was that in order to improve the air quality level in occupied compartments, at low cost, one must define the way the air enters through the doors (on the first floor), passes through the corridors (on the first and second floors), enters through the door's grids and leaves through the windows by extraction fans to the external environment. The air flow rate, with adjusted air flow rate, for each compartment with a long occupation period, was calculated in accordance with the Portuguese standard D.-L. n ${ }^{\circ} 79 / 2006$ of April 4th [22]. In small offices located inside higher compartments the use of air renewed directly from the external environment can be

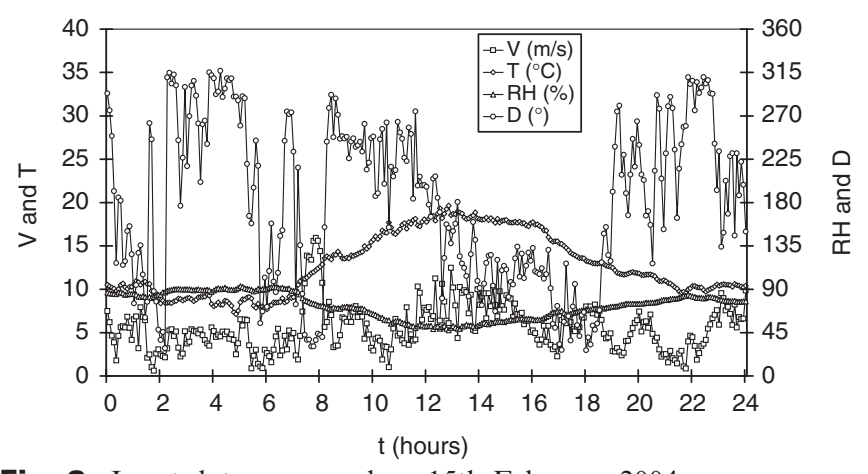

Fig. 2. Input data measured on 15th February 2004.

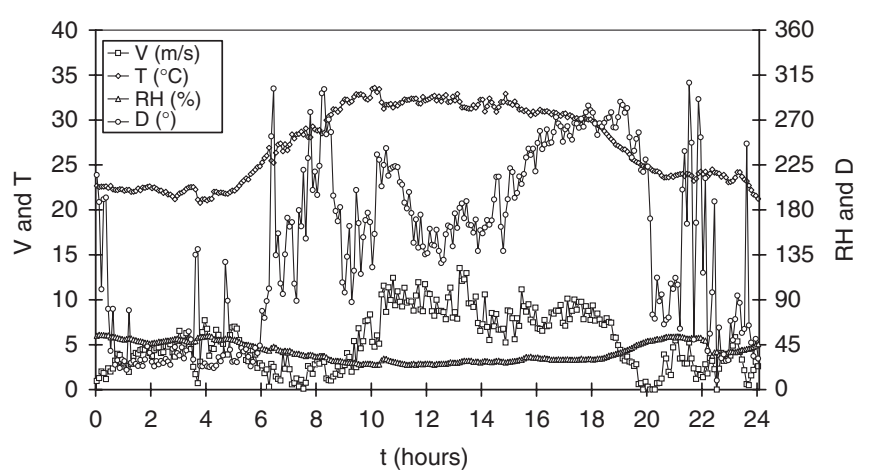

Fig. 3. Input data measured on 12th June 2004.

more efficient. More details were discussed in Conceição and Lúcio [23].

This study was designed to analyze a building built to accommodate around 800 occupants, which had 90-min classes and 15-min breaks. More details about the occupation cycle are given in Conceição and Lúcio [23].

The simulation was conducted as described above and in accordance with ISO 7730 [2] and CR 1752 [3]. That is, comfort conditions for PMV values between -0.5 and 0.5 were considered acceptable.

From Figures 4-11 the evolution of the PMV for the different school building's compartments, in the four orientations, were analyzed. From Figures 4-7, winter conditions are presented, while from Figures 8-11 summer conditions are presented. Figures 4 and 8 are related to layout 1, Figures 5 and 9 are related to layout 2, Figures 6 and 10 are related to layout 3 and Figures 7 and 11 are related to layout 4. All (a) of Figures 4-11 are related to the classrooms, (b) to the offices, (c) to the corridors and (d) to other compartments, namely, buffet, canteen, students' room, teachers' room, library, auditorium, and secretary.

With reference to the figures, the following situations in the classrooms were verified:

- The thermal conditions in classroom nr. 9, under winter conditions, were uncomfortable in the morning 
Table 1. Characteristics of the selected typical rooms

\begin{tabular}{|c|c|c|c|c|c|c|c|}
\hline $\begin{array}{l}\text { Block } \\
\text { identification }\end{array}$ & $\begin{array}{l}\text { Space } \\
\text { number }\end{array}$ & $\begin{array}{c}\text { Space } \\
\text { identification }\end{array}$ & $\begin{array}{l}\text { Space location } \\
\text { in the block }\end{array}$ & $\begin{array}{l}\text { Space location } \\
\text { in the floors levels }\end{array}$ & $\begin{array}{l}\text { Space volume } \\
\left(\mathrm{m}^{3}\right)\end{array}$ & $\begin{array}{l}\text { Windows } \\
\text { location }\end{array}$ & $\begin{array}{c}\text { Windows area } \\
\left(\mathrm{m}^{2}\right)\end{array}$ \\
\hline 3 & 9 & Classroom & Central area & 1 & 402.66 & Two block sides & 28.67 \\
\hline 3 & 17 & Classroom & Central area & 1 & 299.84 & Two block sides & 24.09 \\
\hline 3 & 52 & Classroom & Central area & 2 & 206.27 & One block side & 13.02 \\
\hline 3 & 47 & Classroom & Central area & 2 & 197.39 & One block side & 11.98 \\
\hline 3 & 51 & Classroom & Central area & 2 & 235.33 & One block side & 10.88 \\
\hline 2 & 94 & Classroom & Central area & 2 & 137.40 & One block side & 8.68 \\
\hline 3 & 60 & Office room & Central area & 2 & 60.07 & One block side & 4.34 \\
\hline 1 & 72 & Office room & Central area & 2 & 110.19 & Two block sides & 4.62 \\
\hline 1 & 73 & Office room & Central area & 2 & 142.56 & One block side & 4.34 \\
\hline 1 & 75 & Office room & Central area & 2 & 91.49 & One block side & 4.34 \\
\hline 1 & 76 & Office room & Corner area & 2 & 79.37 & One block side & 4.34 \\
\hline 3 & 10 & Access Corridor & - & 1 and 2 & 311.23 & - & 27.45 \\
\hline 2 & 29 & Access Corridor & - & 1 and 2 & 192.20 & - & 8.88 \\
\hline 2 & 30 & Main Corridor & - & 1 & 493.61 & - & 81.29 \\
\hline 2 & 42 & Access Corridor & - & 1 and 2 & 393.99 & - & 24.76 \\
\hline 2 & 80 & Main Corridor & - & 2 & 353.47 & - & 65.01 \\
\hline 1 & 20 & Buffet & - & 1 & 70.60 & One block side & 22.00 \\
\hline 1 & 26 & Canteen & Corner area & 1 & 623.09 & Two block sides & 44.07 \\
\hline 2 & 40 & Students' room & Corner area & 1 & 611.39 & Two block sides & 32.68 \\
\hline 3 & 59 & Library & Corner area & 2 & 448.15 & Two block sides & 46.11 \\
\hline 1 & 64 & Auditorium & Corner area & 2 & 442.61 & Two block sides & 21.72 \\
\hline 1 & 78 & Secretary & Central area & 2 & 392.94 & One block side & 27.35 \\
\hline 2 & 84 & Teachers' room & Corner area & 2 & 366.62 & Two block sides & 31.62 \\
\hline
\end{tabular}

and comfortable in the afternoon (based on negative PMV values) when the building was oriented as in layouts 1,2, and 3, and comfortable in the morning and afternoon (based on negative PMV values) when the building was oriented as in layout 4 . Under summer conditions this classroom was, in general, thermally uncomfortable in the morning and afternoon, nevertheless, the predicted percentage of dissatisfied people presented the lowest values of all the classrooms;

- In classroom nr. 17, under winter conditions, the PMV index was higher than in classroom nr. 9 in the morning, when the building was oriented in accordance to layouts 1 and 2 and in the morning and afternoon when the building was oriented according to layout 3, nevertheless, this was lower in the other situations. Under summer conditions, except when the building was oriented according to layout 3 , the PMV index was higher than in classroom nr. 9;

- The thermal conditions in classroom nr. 52, under winter conditions, were comfortable in the morning and uncomfortable in the afternoon (based on positive PMV values) when the building was oriented as in layout 1 , uncomfortable in the morning and comfortable in the afternoon when the building was oriented as in layouts 2 and 3 and comfortable in the morning and afternoon (the air temperature increases during the morning to a level maintained in the afternoon) when the building was oriented according to layout 4 . During summer the classroom's thermal conditions were, in general, uncomfortable in the morning and afternoon and the predicted percentage of dissatisfied people presented the highest values when the building was oriented as in layout 4;

- In classroom nr. 47, under winter conditions, the PMV was lower than in classroom nr. 52 when the building was oriented as in layouts 1 (with the exception of the end of the afternoon) and 2 and higher when the building was oriented as in layouts 3 and 4 (with the exception of the start of the morning). Under summer conditions the PMV index was lower than in classroom nr. 52 when the building is oriented according to layouts 2 and 4 and higher when the building was oriented as in layouts 1 and 3;

- The thermal conditions in classroom nr. 51, under winter conditions, were uncomfortable in the morning and comfortable in the afternoon (based on negative PMV values) when the building was oriented according to layout 1 , comfortable in the morning and afternoon (with optimum PMV values) when the building was oriented as in layout 2, comfortable in the morning and afternoon (based on positive PMV values) when the building was oriented as in layout 3 and uncomfortable in the morning and comfortable 

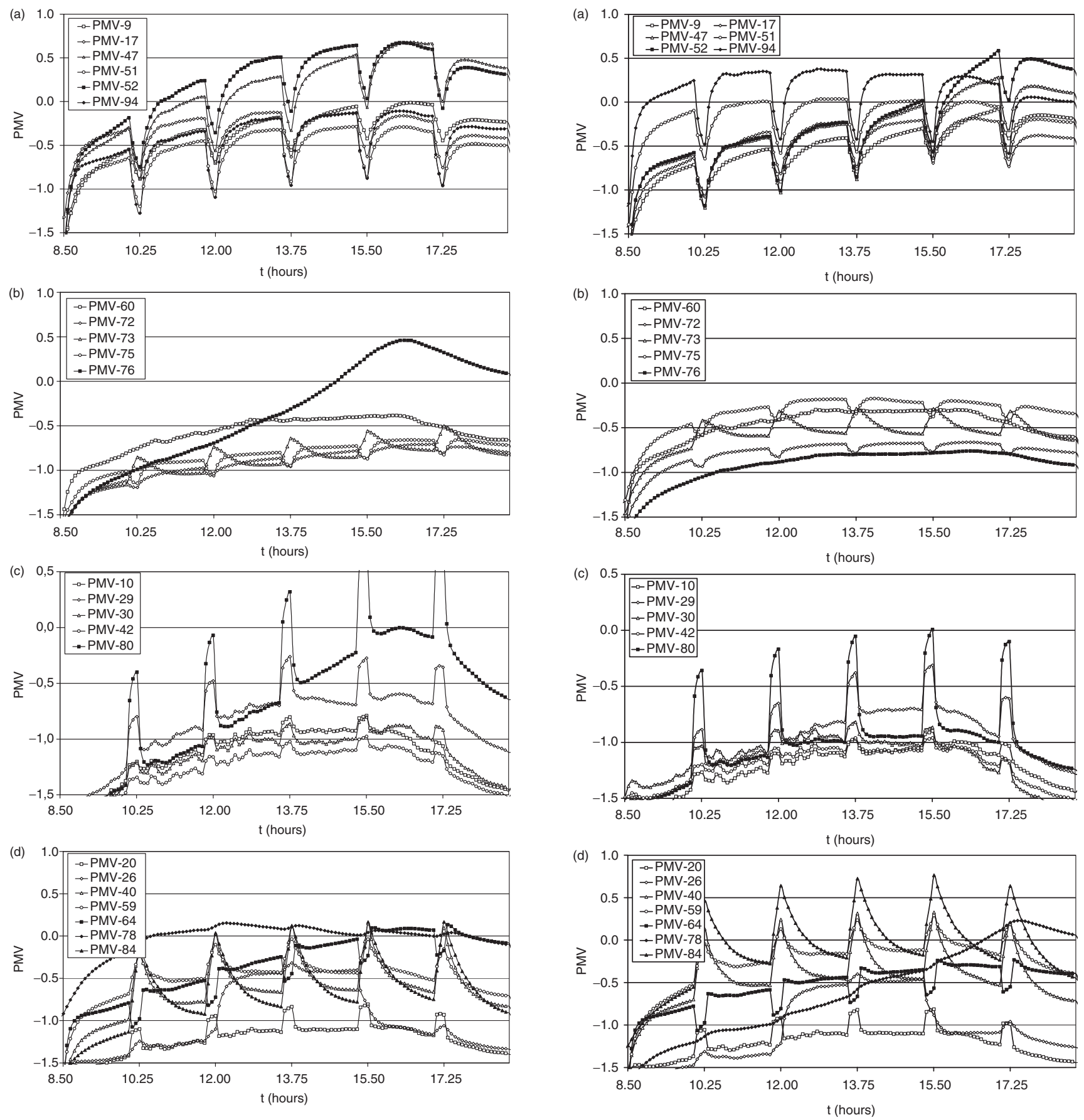

Fig. 5. PMV evolution in Winter conditions for Layout 2.

Fig. 4. PMV evolution in Winter conditions for Layout 1.

in the afternoon when the building was oriented as in layout 4. Under summer conditions the thermal conditions in the classroom were, in general, uncomfortable in the morning and afternoon, and the predicted percentage of dissatisfied people presented the highest values when the building was oriented according to layout 2 ;

- In classroom nr. 94, under winter and summer conditions, in general, the PMV index was higher than in classroom nr. 52.

The analyzed results, for the classrooms, can be used to conclude that:

- Thermal conditions in classrooms with windows turned towards the South (in layout 1 or 3), under 

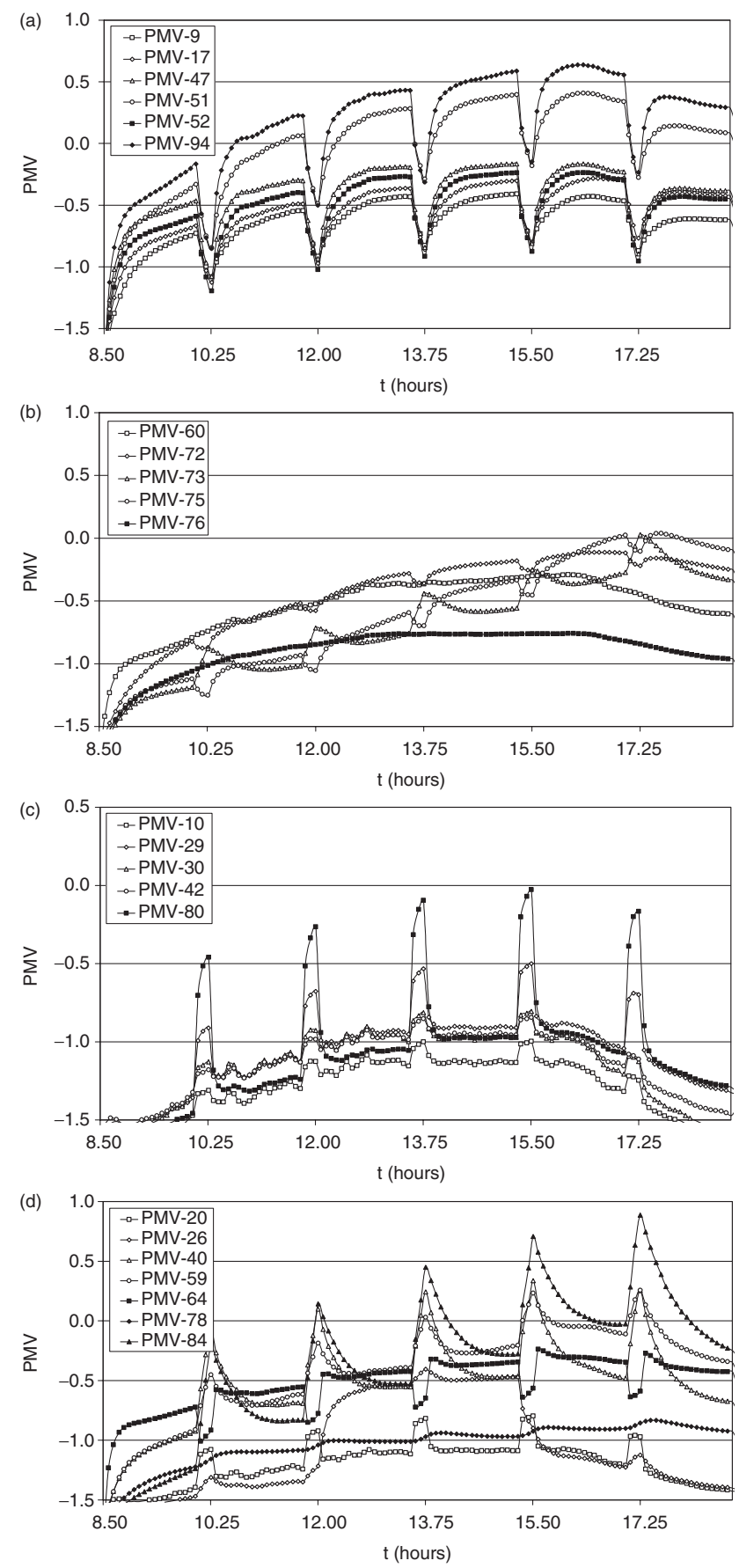

Fig. 6. PMV evolution in Winter conditions for Layout 3.

winter conditions are comfortable in the morning and uncomfortable in the afternoon (based on positive PMV values), while under summer conditions, this set-up produced the highest predicted percentage of dissatisfied people. In order to reduce the air temperature under summer conditions, for example, the use of horizontal shading devices placed above the
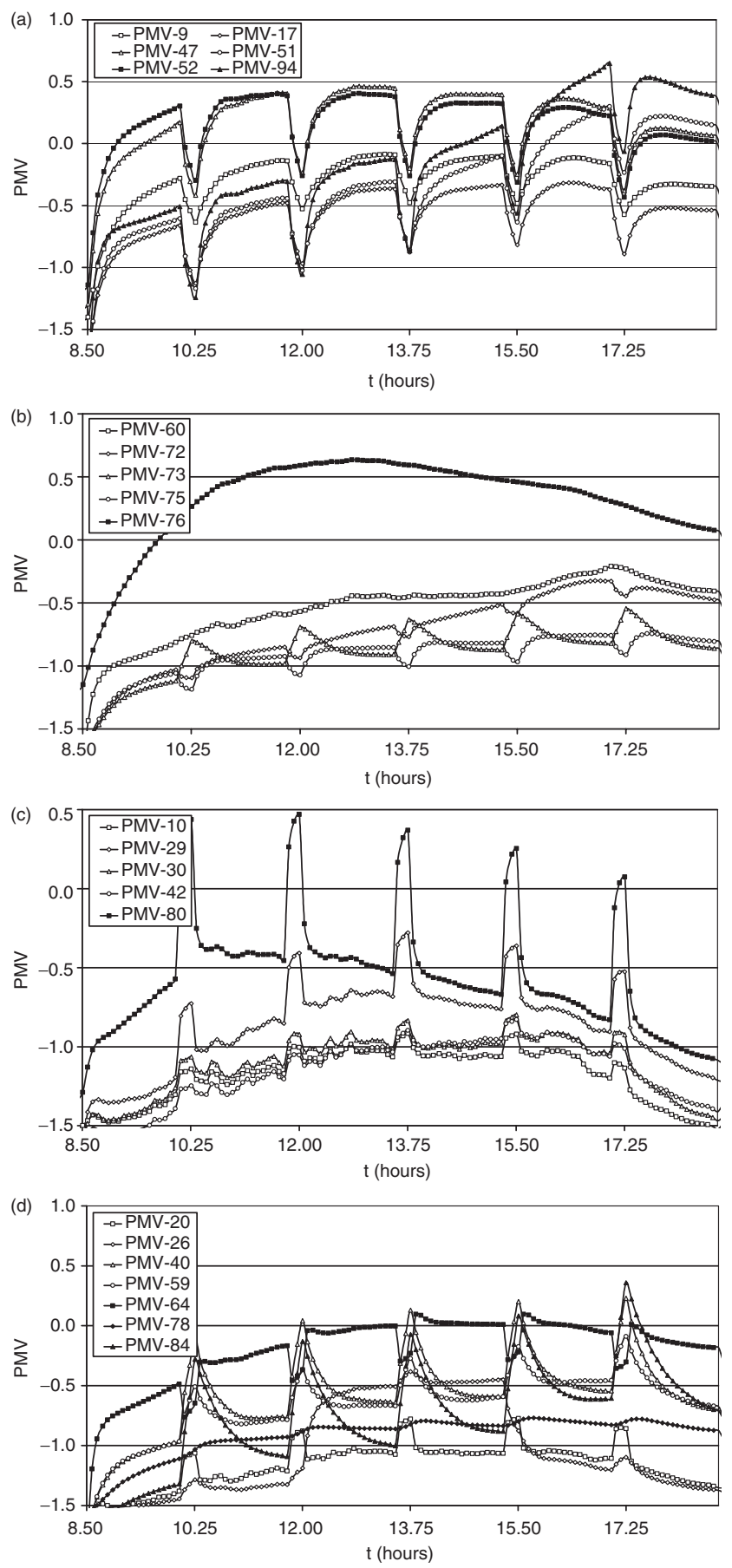

Fig. 7. $P M V$ evolution in Winter conditions for Layout 4.

level of the windows (to reduce incoming solar radiation level, under summer conditions, for windows facing towards the South), air-conditioning systems or underground ducts (using the geothermal heat exchange phenomenon) are suggested;

- The thermal conditions in classrooms with windows facing towards the North (in layouts 1 and 3), under 

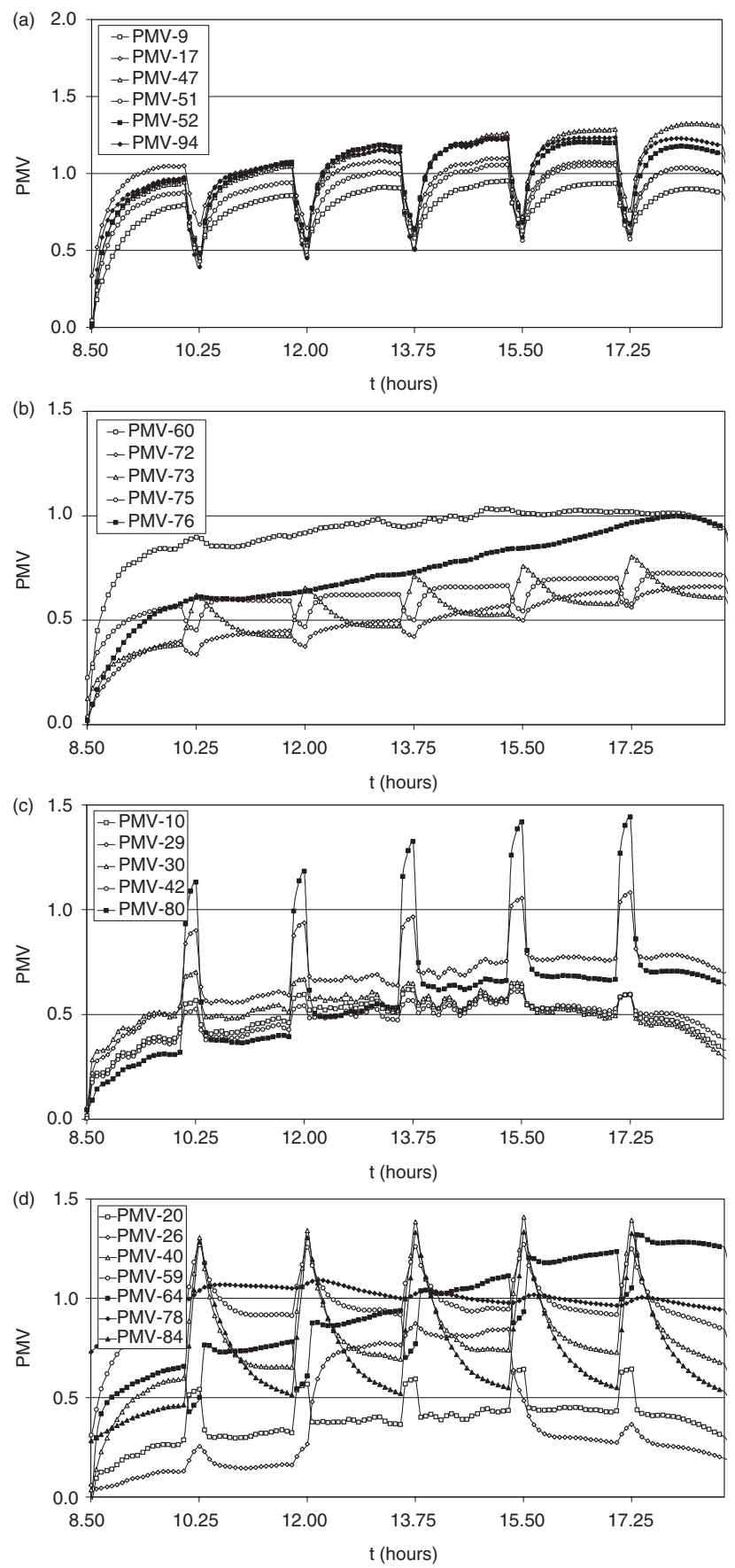

Fig. 8. PMV evolution in Summer conditions for Layout 1 .

winter conditions, in general, are comfortable in the afternoon (based on negative PMV values), nevertheless, if the outside air temperature lowers, they are uncomfortable. The lowest predicted percentage of dissatisfied people (but with levels of discomfort), were found under summer conditions. For a classroom located in the corner of the building the thermal comfort conditions are slightly improved.
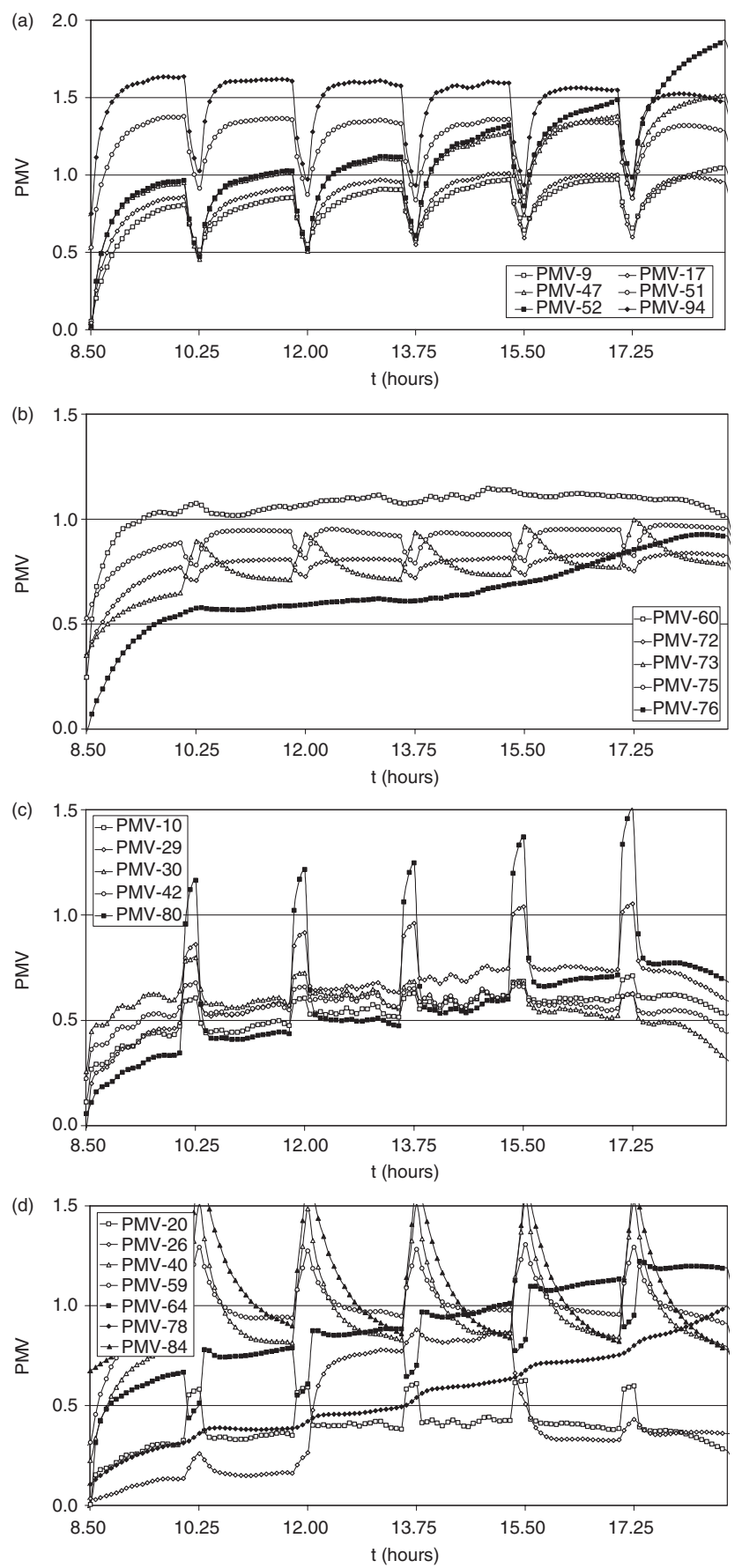

Fig. 9. PMV evolution in Summer conditions for Layout 2.

In order to warm the internal air during winter conditions, as an example, the use of heating systems (see as example Conceição and Lúcio [24]), radiant panels and under-floor heating (see as an example Conceição and Lúcio [25]) or warm air (heated in an air-collector located in the top of the building instead of the roof, see as an example Conceição and Lúcio [24]) are suggested; 

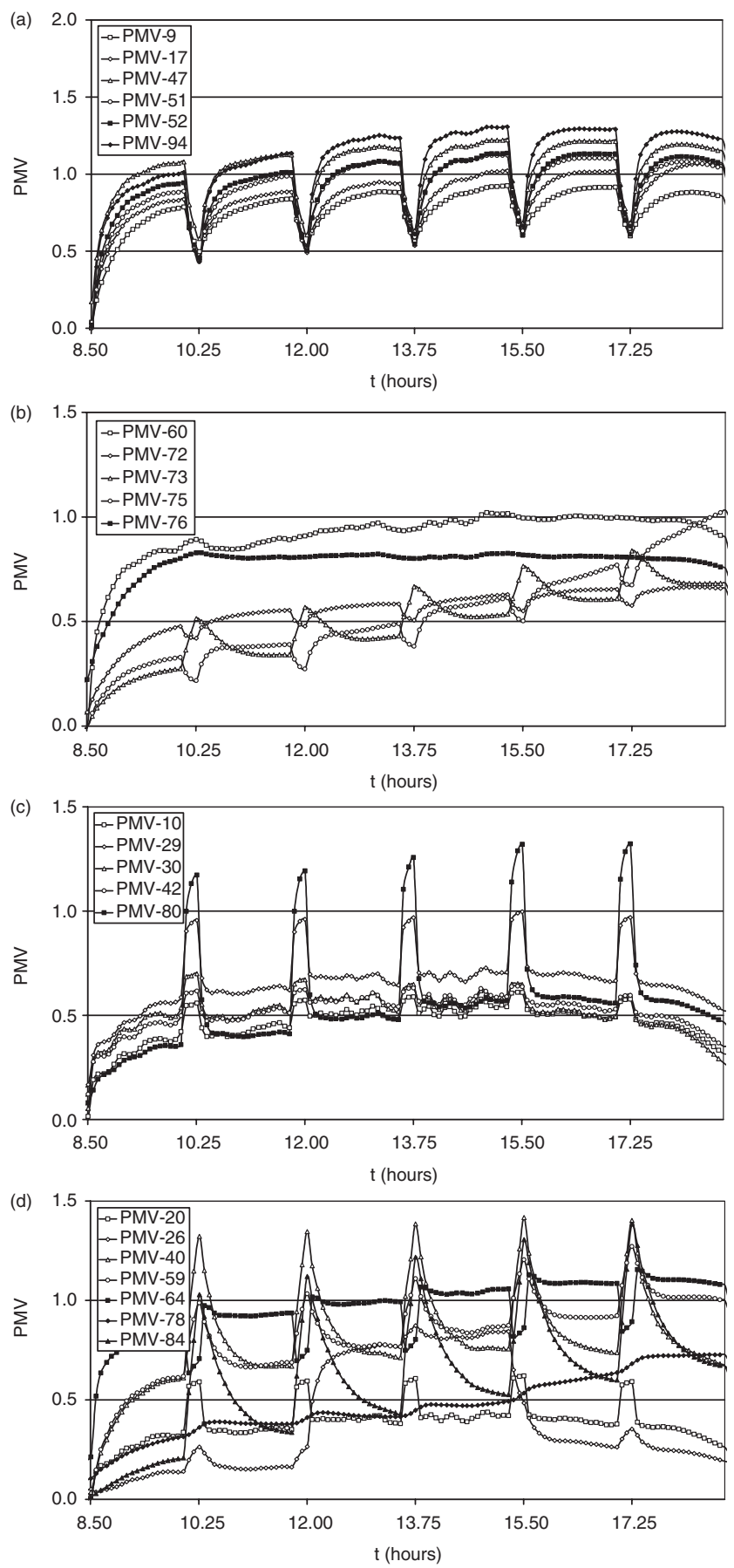

Fig. 10. PMV evolution in Summer conditions for Layout 3.

- The thermal conditions in classrooms with windows facing towards the East, (as in layouts 2 or 4), the air temperature increases in the morning (due to direct solar radiation and the heat released by the occupants), and levels are maintained during the afternoon. Under winter conditions, thermal comfort levels were acceptable, while under summer conditions thermal comfort levels were not acceptable. Under summer
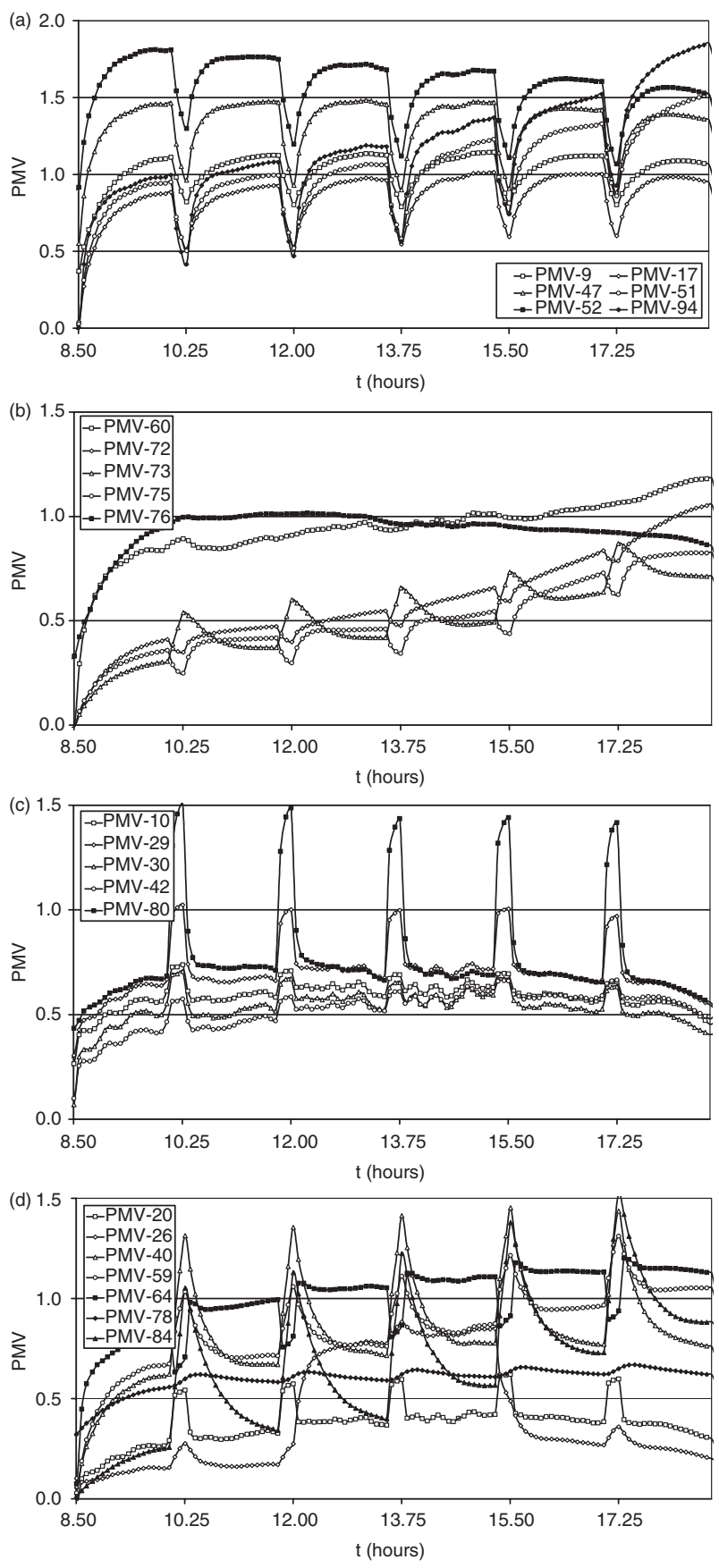

Fig. 11. PMV evolution in Summer conditions for Layout 4.

conditions, in order to reduce the internal air temperature one could, as an example, plant trees outside the building whose foliage could reduce the incoming solar radiation through East facing windows, otherwise air-conditioning systems or underground ducts (using the geothermal heat exchange phenomena) could be used; 
- The thermal conditions in classrooms with windows facing towards the West (as in layouts 2 or 4) under winter conditions, were comfortable in the morning but uncomfortable by the end of the afternoon (based on positive PMV values). Under summer conditions, there were no conditions where thermal comfort was acceptable. As before planting trees could be useful to reduce incoming solar radiation or the other suggestions posed above.
Similar analysis were conducted for offices, corridors, and other compartments. The results can be deduced from the figures.

\section{Acknowledgments}

This research activity is being developed inside a project approved and financed by the FCT, POCI 2010, sponsored by the European Community Fund FEDER.

\section{References}

1 Fanger PO: Thermal Comfort: Analysis and Applications in Environmental Engineering. USA, McGraw-Hill Book Company, 1970.

2 ISO 7730: Moderate Thermal Environments Determination of the PMV and PPD Indices and Specification of the Conditions for Thermal Comfort, International Standard, Suisse, Geneva, 1994.

3 CR 1752: Ventilation for Buildings Design Criteria for the Indoor Environment. CEN (Comité Européen de Normalisation), Brussels, 1998.

4 Fanger PO, Toftum J: Extension of the PMV model to non-air-conditioned buildings in warm climates: Energy Buildings 2002; 34: 533-536.

5 de Dear R, Brager G, Cooper D: Developing an Adaptive Model of Thermal Comfort and Preference. ASHRAE RP-884, 1997.

6 Lazzerini R, Curtis D, Bowen R, Patel M, Ruscisa G, Lazzerini P, Renzio M, Zabot S, Guarino D, Cellie A: ANNEX XV - Energy Efficiency in Schools. Part one, IEA International Energy Agency, 1991.

7 Lazzerini R, Curtis D, Bowen R, Patel M, Ruscisa G, Lazzerini P, Renzio M, Zabot S, Guarino D, Cellie A: ANNEX XV - Energy Efficiency in Schools. Part two, IEA International Energy Agency, 1991.

8 Kolokotroni M, Ge YT, Katsoulas D: Monitoring and modelling indoor air quality and ventilation in classrooms within a purpose-designed naturally ventilated school: Indoor Built Environ 2002; 11(3): 316-326.

9 Conceição EZE, Lúcio M̂MJR, Vicente VDSR: Indoor Thermal Environment in a Classroom Equipped with Air Forced System, REHVA World Congress CLIMA'2007 Wellbeing Indoors, Helsinki, Finland, 10 to 14 of June 2007.

10 Ansaldi R, Corgnati SP, and Filippi M: Comparison Between Thermal Comfort
Predictive Models and Subjective Responses in Italian University Classrooms, REHVA World Congress CLIMA'2007 Wellbeing Indoors, Helsinki, Finland, 10 to 14 of June 2007.

11 Mumovic D, Davies M, Pearson C, Pilmoor G, Ridley I, Altamirano-Medina H, Oreszczyn T: A Comparative Analysis of the Indoor Air Quality and Thermal Comfort in Schools with Natural, Hybrid and Mechanical Ventilation Strategies, REHVA World Congress CLIMA'2007 Wellbeing Indoors, Helsinki, Finland, 10 to 14 of June 2007.

12 Moraes C: The influence of mass advance for altitude tropical climates: establishment of limit conditions for thermal comfort in classrooms, REHVA World Congress CLIMA'2007 Wellbeing Indoors, Helsinki, Finland, 10 to 14 of June 2007.

13 Conceição EZE: Numerical simulation of buildings thermal behavior and human thermal comfort multi-node models, in Proc. of Building Simulation, Netherlands, Eindhoven, August 2003.

14 Conceição EZE, Lúcio MMJR: Numerical Study of Thermal Response of School Buildings in Winter Conditions, in Proc. of RoomVent 2004, Portugal, Coimbra, September 2004

15 Conceição EZE, Lúcio M̂MJR: Numerical simulation of the shading effect verified in school buildings with complex topology in winter conditions, Fourth International Conference on Computational Heat and Mass Transfer, Cachan-Paris, France, 17 to 20 May 2005.

16 Conceição EZE, Lúcio MMJR: Numerical study of thermal response of school buildings in summer conditions, Healthy Buildings 2006, Lisbon, Portugal, 4 to 8 of June 2006
17 Conceição EZE, Lúcio MMJR, Vicente VDSR, Lopes MC, Teixeira ARPA: Comfort Level and Energy Consumption in a New School Building in South of Portugal, REHVA World Congress CLIMA'2007 Wellbeing Indoors, Helsinki, Finland, 10 to 14 of June 2007.

18 Fehlberg E: Low-order Classical Runge-Kutta Formulas with Step Size Control and their Application to Some Heat Transfer Problems. NASA Technical Report 315, 1969.

19 Zhang L, Chow TT, Tsang CF, Chan LS, Fong KF: Effect on air supply temperature on the performance of displacement ventilation (Part I) - thermal comfort: Indoor Built Environ 2005; 14(2): 103-115.

20 Gao NP, Zhang H, Niu JL: Investigating indoor air quality and thermal comfort using a numerical thermal manikin: Indoor Built Environ 2007; 16(1): 7-17.

21 Gao NP, Niu JL: CFD Study of the thermal environment around a human body: Indoor Built Environ 2005; 14(1): 5-16.

22 Decreto-Lei ${ }^{\circ}$ 79: Regulamento dos sistemas energéticos de climatização em edifícios (RSECE) (Regulation of the energy systems and climatisation of buildings), Diário da República, I Série - A, N. 67, April 42006. (in Portuguese).

23 Conceição EZE, Lúcio MMJR: Air quality inside compartments of a school building: air exchange monitoring, evaluation of carbon dioxide and assessment of ventilation strategies: Intl J Vent UK 2006; 5: 259-270.

24 Conceição EZE, Lúcio MMMJ: Thermal study of school buildings in winter conditions: Building Environ 2008; 43(5): 782-792.

25 Conceição EZE, Lúcio MMMR: Evaluation of thermal comfort conditions in a classroom equipped with radiant systems, Healthy Buildings 2006, Lisbon, Portugal, 4 to 8 June 2006. 